Journal of Mathematical Physics, Analysis, Geometry

2014, vol. 10, No. 3, pp. 328-349

\title{
Matrix Riemann-Hilbert Problems and Maxwell-Bloch Equations without Spectral Broadening
}

\author{
V.P. Kotlyarov and E.A. Moskovchenko \\ B. Verkin Institute for Low Temperature Physics and Engineering \\ National Academy of Sciences of Ukraine \\ 47 Lenin Ave., Kharkiv 61103, Ukraine \\ E-mail: kotlyarov@ilt.kharkov.ua
}

Received December 7, 2012, revised March 13, 2014

\begin{abstract}
The Maxwell-Bloch equations have been intensively studied by many authors. The main results are based on the inverse scattering transform and the Marchenko integral equations. However this method is not acceptable for mixed problems. In the paper, we develop a method allowing to linearize mixed problems. It is based on simultaneous spectral analysis of both Lax equations and the matrix Riemann-Hilbert problems. We consider the case of infinitely narrow spectral line, i.e., without spectrum broadening. The proposed matrix Riemann-Hilbert problem can be used for studying temporal/spatial asymptotics of the solutions of Maxwell-Bloch equations by using a nonlinear method of steepest descent.
\end{abstract}

Key words: nonlinear equations, Riemann-Hilbert problem, the steepest descent method, asymptotics.

Mathematics Subject Classification 2010: 37K15, 35Q15, 35B40.

\section{Introduction}

The Maxwell-Bloch (MB) equations became well known after Lamb [1-4]. In [5], Ablowitz, Kaup and Newell proposed the inverse scattering transform (IST) to the Maxwell-Bloch equations for studying a physical phenomenon known as self-induced transparency. A description of general solutions to the MB equations and their classifying was done by Gabitov, Zakharov and Mikhailov in [6]. All the authors used the IST method based on the Marchenko integral equations. In particular, in [6], the authors gave an approximate solution of the mixed problem to the MB equations in the domain $x, t \in(0, L) \times(0, \infty)$. They also emphasized that the IST method is not adopted for mixed problems. In this paper, we develop a method allowing to linearize mixed problems in the case of infinitely narrow

(C) V.P. Kotlyarov and E.A. Moskovchenko, 2014 
spectral line, i.e., without spectral broadening. It is based on the simultaneous spectral analysis of both the Lax equation, associated with the Maxwell-Bloch equations, and the matrix Riemann-Hilbert problems. The proposed matrix $\mathrm{RH}$ problems will be useful for studying the long time/long distance $\left(x \in \mathbb{R}_{+}\right)$ asymptotic behavior of solutions to the MB equations by using the nonlinear method of steepest descent as, for example, in [7] and [8].

We consider the Maxwell-Bloch equations in the form given in [6]:

$$
\begin{gathered}
\frac{\partial \mathcal{E}}{\partial t}+\frac{\partial \mathcal{E}}{\partial x}=\langle\rho\rangle, \\
\frac{\partial \rho}{\partial t}+2 \mathrm{i} \lambda \rho=\mathcal{N} \mathcal{E}, \\
\frac{\partial \mathcal{N}}{\partial t}=-\frac{1}{2}\left(\mathcal{E}^{*} \rho+\mathcal{E} \rho^{*}\right) .
\end{gathered}
$$

Here $*$ denotes the complex conjugation, $\mathcal{E}=\mathcal{E}(t, x)$ is a complex valued function of the space variable $x$ and the time $t, \rho=\rho(t, x, \lambda)$ and $\mathcal{N}(t, x, \lambda)$ are complex valued and real functions of $t, x$ and the spectral parameter $\lambda$. The angular brackets \langle\rangle mean averaging on $\lambda$ with a given weight function $n(\lambda)>0$,

$$
\langle\rho\rangle=\int_{-\infty}^{\infty} \rho(t, x, \lambda) n(\lambda) d \lambda, \quad \int_{-\infty}^{\infty} n(\lambda) d \lambda=1 .
$$

Equations (1)-(4) can be found in a number of physical models. One of the most important is a model of the propagation of electromagnetic waves in a medium with distributed two-level atoms. In particular, there are models of self-induced transparency [5, 9], and quantum laser amplifier [10, 11]. For these models, $\mathcal{E}(t, x)$ is the complex valued envelope of electromagnetic wave of a fixed polarization, $\mathcal{N}(t, x, \lambda)$ and $\rho(t, x, \lambda)$ are entries of the density matrix of the atom subsystem

$$
\hat{\rho}(t, x, \lambda)=\left(\begin{array}{cc}
\mathcal{N}(t, x, \lambda) & \rho(t, x, \lambda) \\
\rho^{*}(t, x, \lambda) & -\mathcal{N}(t, x, \lambda)
\end{array}\right) .
$$

The parameter $\lambda$ denotes a deviation of the passage frequency from its mean value, and the function $n(\lambda)$ characterizes the inhomogeneous broadening, i.e., it describes the form of a spectral line. For short reviews on the MB equations see $[5,6,9]$.

We restrict ourselves to the case of the infinitely narrow spectral line where $n(\lambda)=\delta(\lambda)$. Then $\langle\rho\rangle=\rho$, and (1)-(3) takes the form

$$
\frac{\partial \mathcal{E}}{\partial t}+\frac{\partial \mathcal{E}}{\partial x}=\rho, \quad \frac{\partial \rho}{\partial t}=\mathcal{N} \mathcal{E}, \quad \frac{\partial \mathcal{N}}{\partial t}=-\frac{1}{2}\left(\mathcal{E}^{*} \rho+\mathcal{E} \rho^{*}\right) .
$$


The mixed problem for the Maxwell-Bloch equations is defined by the following initial and boundary conditions:

$$
\mathcal{E}(0, x)=\mathcal{E}_{0}(x), \quad \rho(0, x)=\rho_{0}(x), \quad \mathcal{N}(0, x)=\mathcal{N}_{0}(x), \quad \mathcal{E}(t, 0)=\mathcal{E}_{1}(t),
$$

where $x \in(0, L)(L \leq \infty)$ and $t \in \mathbb{R}_{+}$. The function $\mathcal{E}_{1}(t)$ is a Schwartz type function (smooth and fast decreasing at infinity). The functions $\mathcal{E}_{0}(x), \rho_{0}(x)$, $\mathcal{N}_{0}(x)$ are smooth or Schwartz type functions if $x \in \mathbb{R}_{+}$. If one deals with a solution on the whole $t$-line, then the input pulse $\mathcal{E}_{1}(t)$ should be given for $t \in \mathbb{R}$, and the functions $\rho(t, x), \mathcal{N}(t, x)$ should be given as $t \rightarrow-\infty$.

The functions $\rho(t, x), \mathcal{N}(t, x)$ are not independent. Indeed, Eqs. (2) and (3) give

$$
\frac{\partial}{\partial t}\left(|\rho(t, x)|^{2}+\mathcal{N}(t, x)\right)=0
$$

and we put

$$
|\rho(t, x)|^{2}+\mathcal{N}(t, x) \equiv 1 .
$$

Thus we must define $\rho(0, x)$ and

$$
\mathcal{N}(0, x)=\mp \sqrt{1-|\rho(0, x)|^{2}} .
$$

We choose the sign "minus" to have a stable medium, the so-called attenuator ( for example, in the model of self-induced transparency). The sign "plus" corresponds to an unstable medium, i.e., to a quantum laser amplifier.

Assuming that the medium is stable, we consider the functions $\mathcal{E}(t, x), \rho(t, x)$ and $\mathcal{N}(t, x)$ which satisfy the MB Eqs. (6) in the domain $x, t \in(0, L) \times(0, \infty)$. We develop the IST method in the form of the matrix Riemann-Hilbert problem in the complex $z$-plane and give an integral representation for $\mathcal{E}(t, x)$ through the solution of a singular integral equation which is equivalent to the matrix $\mathrm{RH}$ problem. This RH problem is produced by spectral functions defined via given initial and boundary conditions for MB equations. Further, we give a formulation of a more general matrix $\mathrm{RH}$ problem which has a unique solution. We prove that the $\mathrm{RH}$ problem generates a compatibility system of differential equations which is the AKNS linear Eqs. [9] for the MB equations without spectral broadening. Thus this RH problem generates different solutions to the MB equations. Among them there are the solutions on the whole $t$-axis, the solutions of the mixed problem in the quarter $t x$-plane with vanishing at infinity or some periodic in $t$ input pulse $\mathcal{E}(t, 0)$.

Our approach differs from that considered in [12] for the Goursat problem to the MB equations where a linear (but complicated) "evolution" in $x$ takes place for scattering data. We develop the approach of simultaneous spectral analysis proposed in [13-16] and in [17-20], and prove that the mixed problem is completely linearizable by the appropriate matrix $\mathrm{RH}$ problem. 


\section{Basic Solutions of the Ablowitz-Kaup-Newel-Segur Linear Equations}

We use the simultaneous spectral analysis of the linear $t$-equation

$$
\begin{aligned}
\Phi_{t}+\mathrm{i} \lambda \sigma_{3} \Phi & =-H(t, x) \Phi \\
\sigma_{3}=\left(\begin{array}{cc}
1 & 0 \\
0 & -1
\end{array}\right), \quad H(t, x) & =\frac{1}{2}\left(\begin{array}{cc}
0 & \mathcal{E}(t, x) \\
-\mathcal{E}^{*}(t, x) & 0
\end{array}\right)
\end{aligned}
$$

and the linear $x$-equation

$$
\begin{gathered}
\Phi_{x}-\mathrm{i}\left(\lambda \sigma_{3}+\frac{F(t, x))}{4 \lambda}\right) \Phi=H(t, x) \Phi, \\
F(t, x)=\left(\begin{array}{cc}
\mathcal{N}(t, x) & \rho(t, x) \\
\rho^{*}(t, x) & -\mathcal{N}(t, x)
\end{array}\right) .
\end{gathered}
$$

Here $\Phi(t, x, \lambda)$ is a $2 \times 2$ matrix-valued function and $\lambda \in \mathbb{R}$ is a parameter. It is easy to verify that the over-determined system of differential Eqs. (8), (9) (AKNS system of equations [9]) is compatible if and only if the functions $\mathcal{E}(t, x)$, $\rho(t, x$,$) and \mathcal{N}(t, x)$ satisfy the MB Eqs. (6).

Let us rewrite equations (8) and (9) in the form:

$$
\begin{aligned}
& W_{t}=U(t, x, \lambda) W, \\
& W_{x}=V(t, x, \lambda) W,
\end{aligned}
$$

where $U$ and $V$ are the matrices:

$$
\begin{aligned}
& U(t, x, \lambda)=-\left(\mathrm{i} \lambda \sigma_{3}+H(t, x)\right), \\
& V(t, x, \lambda)=\mathrm{i} \lambda \sigma_{3}+H(t, x)+\frac{\mathrm{i} F(t, x)}{4 \lambda} .
\end{aligned}
$$

Lemma 2.1. Let Eqs. (10) and (11) be compatible for all $t, x, \lambda \in \mathbb{R}$. Let $W(t, x, \lambda)$ be a matrix satisfying the t-equation (10) for all $x$ (the $x$-equation (11) for all $t)$. Assume that $W\left(t_{0}, x, \lambda\right)$ satisfies the $x$-equation (11) for some $t=t_{0} \leq \infty$ (the $t$-equation (10) for some $\left.x=x_{0} \leq \infty\right)$. Then $W(t, x, \lambda)$ satisfies the $x$-equation (11) for all $t$ (satisfies the $t$-equation (10) for all $x$ ).

P r o o f. See, for example, in [17] (Lemma 2.1). 


\subsection{The problem on whole $t$-line}

Let $\mathcal{E}(t, x), \rho(t, x)$ and $\mathcal{N}(t, x)$ be a smooth solution to the MB Eqs. (6). We suppose additionally that $\mathcal{E}(t, x)$ and $\rho(t, x)$ are fast decreasing as $t \rightarrow-\infty$ (hence $\mathcal{N}(t, x) \rightarrow-1$ as $t \rightarrow-\infty)$. This problem was first studied in [5] by using the inverse scattering transform and the Marchenko integral equation. Below we give the formulation of the corresponding matrix Riemann-Hilbert problem.

Let $Y(t, x, \lambda)$ be a product of the matrices

$$
Y(t, x, \lambda)=W(t, x, \lambda) \Phi(t, \lambda),
$$

where $W(t, x, \lambda)$ satisfies the $x$-equation for all $t$ and $W(t, 0, \lambda)=I$, and $\Phi(t, \lambda)$ satisfies the $t$-equation for $x=0$ under the initial condition $\lim _{t \rightarrow \infty} \Phi(t, \lambda) e^{\mathrm{i} \lambda t \sigma_{3}}=I$. Then, due to Lemma 2.1, the matrix $Y(t, x, \lambda)$ is a compatible solution of the Ablowitz-Kaup-Newel-Segur (AKNS) system of equations (8)-(9).

Let $Z(t, x, \lambda)$ be a compatible solution of the AKNS system of equations (8)(9) such that

$$
Z(t, x, \lambda)=\Psi(t, x, \lambda) W_{\infty}(x, \lambda),
$$

where $\Psi(t, x, \lambda)$ satisfies the $t$-equation for all $0 \leq x \leq L \leq \infty$ under the initial condition $\lim _{t \rightarrow-\infty} \Psi(t, x, \lambda) e^{\mathrm{i} \lambda t \sigma_{3}}=I$, and $W_{\infty}(x, \lambda)$ satisfies the $x$-equation whose coefficients are constant matrices as $t \rightarrow-\infty$. It is normalized by the initial condition $W_{\infty}(0, \lambda) \equiv I$. It is easy to see that $W(t, x, \lambda)$ is the fundamental solution of the $x$-equation with fixed $t \in \mathbb{R}$. The matrices $\Phi(t, \lambda)$ and $\Psi(t, x, \lambda)$ are the Jost solutions of the $t$-equation with any fixed $x$.

Lemma 2.2. Let $\mathcal{E}(t, x), \rho(t, x)$ and $\mathcal{N}(t, x)$ be smooth solutions to the $M B$ equations (6) such that $\mathcal{E}(t, x)$ and $\rho(t, x)$ are fast decreasing as $t \rightarrow-\infty$. Let $\mathcal{E}(t, 0)=\mathcal{E}_{1}(t)$ be smooth and fast decreasing as $|t| \rightarrow \infty$. Then the Jost solutions $\Phi(t, \lambda)$ and $\Psi(t, x, \lambda)$ have the integral representations:

$$
\begin{array}{cc}
\Phi(t, \lambda)=e^{-\mathrm{i} \lambda t \sigma_{3}}+\int_{t}^{\infty} K^{+}(t, \tau, 0) e^{-\mathrm{i} \lambda \tau \sigma_{3}} \mathrm{~d} \tau, & \operatorname{Im} \lambda=0 . \\
\Psi(t, x, \lambda)=e^{-\mathrm{i} \lambda t \sigma_{3}}+\int_{-\infty}^{t} K^{-}(t, \tau, x) e^{-\mathrm{i} \lambda \tau \sigma_{3}} \mathrm{~d} \tau, & \operatorname{Im} \lambda=0 .
\end{array}
$$

The kernels $K^{ \pm}(t, \tau,$.$) satisfy the symmetry condition$ $K^{ \pm *}(t, \tau,)=.\Lambda K^{ \pm}(t, \tau,.) \Lambda^{-1}$ with the matrix $\Lambda=\left(\begin{array}{cc}0 & 1 \\ -1 & 0\end{array}\right)$, and

$$
\left[\sigma_{3}, K^{ \pm}(t, t, .)\right]= \pm \sigma_{3} H(t, .) .
$$

The kernels $K^{ \pm}(t, \tau,$.$) are smooth and fast decreasing as t+\tau \rightarrow \pm \infty$. 
The proof of this lemma is well known (cf. [21]). It is also well known that the vectors $\Phi[1](t, \lambda)$ and $\Phi[2](t, \lambda)$ of the matrix $\Phi(t, \lambda)=(\Phi[1](t, \lambda), \Phi[2](t, \lambda))$ have the analytic continuations $\Phi[1](t, z)$ and $\Phi[2](t, z)$ to the lower and upper half-planes of the complex $z$-plane $(z=\lambda+\mathrm{i} \nu)$, respectively. The vector columns $\Psi[1](t, x, \lambda)$ and $\Psi[2](t, x, \lambda)$ of the matrix $\Psi(t, x, \lambda)=(\Psi[1](t, x, \lambda), \Psi[2](t, x, \lambda))$ have the analytic continuations $\Psi[1](t, x, z)$ and $\Psi[2](t, x, z)$ to the upper and lower half-planes of the complex plane.

If $H(t, x) \equiv 0$ and $F(t, x) \equiv-\sigma_{3}$, then the $x$-equation has an exact solution $e^{\mathrm{i} x \eta(\lambda) \sigma_{3}}$, where $\eta(\lambda)=\lambda-\frac{1}{4 \lambda}$. Taking this into account, we find that the function $W_{\infty}(x, \lambda)=e^{\mathrm{i} \eta(\lambda) x \sigma_{3}}$, and the function $\hat{W}(t, x, \lambda)=e^{-\mathrm{i} \eta(\lambda) x \sigma_{3}} W(t, x, \lambda)$ must satisfy the integral equation

$$
\left.\hat{W}(t, x, \lambda)=I+\int_{0}^{x} e^{-\mathrm{i} y \eta(\lambda) \sigma_{3}}\left(H(t, y)+\mathrm{i}\left(F(t, y)+\sigma_{3}\right) / 4 \lambda\right)\right) e^{\mathrm{i} y \eta(\lambda) \sigma_{3}} \hat{W}(t, y, \lambda) \mathrm{d} y .
$$

The equation yields that $W(t, x, \lambda)$ has an analytic continuation to the punctured complex plane $\mathbb{C} \backslash\{0\}$.

Lemma 2.3. Let $\mathcal{E}(t, x), \mathcal{N}(t, x), \rho(t, x)$ be smooth functions. Then the solution $W(t, x, \lambda)$ can be represented in the form:

$$
W(t, x, \lambda)=e^{\mathrm{i} \eta(\lambda) x \sigma_{3}} \hat{W}(t, x, \lambda),
$$

where $\hat{W}(t, x, \lambda)$ is the unique solution of the Volterra integral equation (16). The solution $W(t, x, \lambda)$ is smooth in $t$ and $x$, and it has an analytic continuation $W(t, x, z)$ where $z=\lambda+\mathrm{i} \nu \in \mathbb{C} \backslash\{0\}$. Moreover, the matrix $W(t, x, z) e^{-\mathrm{i} \eta(z) x}$ is continuous, bounded in $\mathbb{C}_{-} \cup \mathbb{R}$ and has the asymptotics

$$
W(t, x, z) e^{-\mathrm{i} \eta(z) x}=\left(\begin{array}{cc}
1 & 0 \\
0 & e^{-2 \mathrm{i} z x}
\end{array}\right)+O\left(z^{-1}\right), \quad \operatorname{Im} z \leq 0, \quad z \rightarrow \infty,
$$

and the matrix $W(t, x, z) e^{\mathrm{i} \eta(z) x}$ is continuous, bounded in $\mathbb{C}_{+} \cup \mathbb{R}$ and has the asymptotics

$$
W(t, x, z) e^{\mathrm{i} \eta(z) x}=\left(\begin{array}{cc}
e^{2 \mathrm{i} z x} & 0 \\
0 & 1
\end{array}\right)+O\left(z^{-1}\right), \quad \operatorname{Im} z \geq 0, \quad z \rightarrow \infty,
$$

where the symbol $\mathrm{O}($.$) means a matrix whose entries have the indicated order.$

P r o of. The solvability of the Volterra integral Eq. (16) and the smoothness of the solution with respect to $t$ and $x$ can be easily proved by using the method of successive approximations. Since the left-hand side

$$
\mathrm{i} z \sigma_{3}+H(t, x)+\mathrm{i}\left(F(t, x)+\sigma_{3}\right) / 4 z
$$


of the $x$-equation is analytic for $z \neq 0$, the matrix $W(t, x, \lambda)$ has an analytic continuation for $z=\lambda+\mathrm{i} \nu \in \mathbb{C} \backslash\{0\}$, which we denote as $W(t, x, z)$. Thus the matrix $W(t, x, z) e^{-\mathrm{i} \eta(z) x}\left(W(t, x, z) e^{\mathrm{i} \eta(z) x}\right)$ is analytic, continuous for $z \neq 0$ in $\mathbb{C}_{-} \cup \mathbb{R}\left(\mathbb{C}_{+} \cup \mathbb{R}\right)$ and has the above asymptotics. Moreover, they are bounded as $z \rightarrow 0$ and, hence, we find (determine) that they are continuous also at the point $z=0$.

Formulas (12), (14), (17) and Lemmas 2.2, 2.3 imply the following properties of the matrix $Y(t, x, \lambda)=(Y[1](t, x, \lambda) \quad Y[2](t, x, \lambda))$ :

1) $Y(t, x, \lambda)(\lambda \neq 0)$ satisfies the $t$ - and $x$-equations (8)-(9);

2) $Y(t, x, \lambda)=\Lambda Y^{*}(t, x, \lambda) \Lambda^{-1}, \lambda \in \mathbb{R} \backslash\{0\}$, where $\Lambda=\left(\begin{array}{cc}0 & 1 \\ -1 & 0\end{array}\right)$;

3) $\operatorname{det} Y(t, x, \lambda) \equiv 1, \quad \lambda \in \mathbb{R} \backslash\{0\}$;

4) the map $(x, t) \longmapsto Y(t, x, \lambda)(\lambda \neq 0)$ is smooth in $t$ and $x$;

$5)$ the vector column $Y[1](t, x, \lambda)$ has the analytic continuation $Y[1](t, x, z)$ for $z \in \mathbb{C}_{-}$, and $Y[1](t, x, z) e^{\mathrm{i} z t-\mathrm{i} x \eta(z)}$ is continuous in $z \in \mathbb{C}_{-} \cup \mathbb{R}$, and

$$
Y[1](t, x, z) e^{\mathrm{i} z t-\mathrm{i} x \eta(z)}=\left(\begin{array}{l}
1 \\
0
\end{array}\right)+O\left(z^{-1}\right), \quad z \rightarrow \infty ;
$$

6) the vector column $Y[2](t, x, \lambda)$ has the analytic continuation $Y[2](t, x, z)$ for $z \in \mathbb{C}_{+}$, and $Y[2](t, x, z) e^{-\mathrm{i} z t+\mathrm{i} x \eta(z)}$ is continuous in $z \in \mathbb{C}_{+} \cup \mathbb{R}$, and

$$
Y[2](t, x, z) e^{-\mathrm{i} z t+\mathrm{i} x \eta(z)}=\left(\begin{array}{l}
1 \\
0
\end{array}\right)+O\left(z^{-1}\right), \quad z \rightarrow \infty .
$$

These asymptotics follow from the formulas:

$$
\begin{gathered}
\Phi[1](t, z) e^{\mathrm{i} z t}=\left(\begin{array}{l}
1 \\
0
\end{array}\right)+O\left(z^{-1}\right), \quad \operatorname{Im} z<0, \quad z \rightarrow \infty, \\
\Phi[2](t, z) e^{-\mathrm{i} z t}=\left(\begin{array}{l}
0 \\
1
\end{array}\right)+O\left(z^{-1}\right), \quad \operatorname{Im} z>0, \quad z \rightarrow \infty,
\end{gathered}
$$

and

$$
\begin{gathered}
W[1](t, x, z) e^{-\mathrm{i} \eta(z) x}=\left(\begin{array}{l}
1 \\
0
\end{array}\right)+O\left(z^{-1}\right), \quad \operatorname{Im} z<0, \quad z \rightarrow \infty, \\
W[2](t, x, z) e^{\mathrm{i} \eta(z) x}=\left(\begin{array}{l}
0 \\
1
\end{array}\right)+O\left(z^{-1}\right), \quad \operatorname{Im} z>0, \quad z \rightarrow \infty .
\end{gathered}
$$

Taking into account that $W_{\infty}(x, \lambda)=e^{\mathrm{i} \eta(\lambda) x \sigma_{3}}$ and Eqs. (13), (15), we find that:

1) $Z(t, x, \lambda)(\lambda \neq 0)$ satisfies the $t$ - and $x$-equations $(8)-(9)$;

2) $Z(t, x, \lambda)=\Lambda Z^{*}(t, x, \lambda) \Lambda^{-1}, \lambda \in \mathbb{R} \backslash\{0\}$; 
3) $\operatorname{det} Z(t, x, \lambda) \equiv 1, \quad \lambda \in \mathbb{R} \backslash\{0\}$;

4) the map $(x, t) \longmapsto Z(t, x, \lambda)(\lambda \neq 0)$ is smooth in $t$ and $x$;

$5)$ the vector column $Z[1](t, x, \lambda)$ has the analytic continuation $Z[1](t, x, z)$ for $z \in \mathbb{C}_{+}$, and $Z[1](t, x, z) e^{\mathrm{i} z t-\mathrm{i} x \eta(z)}$ is continuous in $z \in \mathbb{C}_{+} \cup \mathbb{R}$, and

$$
Z[1](t, x, z) e^{\mathrm{i} z t-\mathrm{i} x \eta(z)}=\left(\begin{array}{l}
1 \\
0
\end{array}\right)+O\left(z^{-1}\right), \quad z \rightarrow \infty ;
$$

6) the vector column $Z[2](t, x, \lambda)$ has the analytic continuation $Z[2](t, x, z)$ for $z \in \mathbb{C}_{-}$, and $Z[2](t, x, z) e^{-\mathrm{i} z t+\mathrm{i} x \eta(z)}$ is continuous in $z \in \mathbb{C}_{-} \cup \mathbb{R}$, and

$$
Z[2](t, x, z) e^{-\mathrm{i} z t+\mathrm{i} x \eta(z)}=\left(\begin{array}{l}
1 \\
0
\end{array}\right)+O\left(z^{-1}\right), \quad z \rightarrow \infty .
$$

Since the matrices $Y(t, x, \lambda)$ and $Z(t, x, \lambda)$ are the solutions of the $t$ - and $x$-equations (8)-(9), they are linearly dependent. Thus, there exists a transition matrix $T(\lambda)$, independent of $x$ and $t$, such that

$$
Y(t, x, \lambda)=Z(t, x, \lambda) T(\lambda)
$$

The transition matrix is equal to

$$
T(\lambda)=Z^{-1}(0,0, \lambda) Y(0,0, \lambda)=\Psi^{-1}(0,0, \lambda) \Phi(0, \lambda)
$$

and, hence, $T(\lambda)=\Lambda T^{*}(\lambda) \Lambda^{-1}$, i.e., $T(\lambda)$ has the form

$$
T(\lambda)=\left(\begin{array}{cc}
\bar{a}(\lambda) & b(\lambda) \\
-\bar{b}(\lambda) & a(\lambda)
\end{array}\right), \quad \bar{a}(\lambda)=a^{*}(\lambda), \quad \bar{b}(\lambda)=b^{*}(\lambda) .
$$

The scattering relation (18) can be written in the following form:

$$
\begin{array}{rlrl}
Y[1](t, x, \lambda) & =\bar{a}(\lambda) Z[1](t, x, \lambda)-\bar{b}(\lambda) Z[2](t, x, \lambda), & & \lambda \in \mathbb{R}, \\
Y[2](t, x, \lambda)=a(\lambda) Z[2](t, x, \lambda)+b(\lambda) Z[1](t, x, \lambda), & & \lambda \in \mathbb{R} .
\end{array}
$$

These relations give

$$
a(\lambda)=\operatorname{det}(Z[1](t, x, \lambda), Y[2](t, x, \lambda)), \quad b(\lambda)=\operatorname{det}(Y[2](t, x, \lambda), Z[2](t, x, \lambda)) .
$$

The matrices $\Psi(0,0, \lambda)$ and $\Phi(0, \lambda)$ have the form

$$
\left(\begin{array}{cc}
\alpha(\lambda) & -\bar{\beta}(\lambda) \\
\beta(\lambda) & \bar{\alpha}(\lambda)
\end{array}\right) \text { and } \quad\left(\begin{array}{cc}
\bar{A}(\lambda) & B(\lambda) \\
-\bar{B}(\lambda) & A(\lambda)
\end{array}\right),
$$

respectively. The functions $\alpha(\lambda), \beta(\lambda)$ and $A(\lambda), B(\lambda)$ have analytic continuations in $\mathbb{C}_{+}$and $\bar{\alpha}(z)=\alpha^{*}\left(z^{*}\right), \bar{\beta}(z)=\beta^{*}\left(z^{*}\right)$, and $\bar{A}(z)=A^{*}\left(z^{*}\right), \bar{B}(z)=B^{*}\left(z^{*}\right)$ are analytic in $\mathbb{C}_{-}$. Thus we have

$$
a(\lambda)=\alpha(\lambda) A(\lambda)-\beta(\lambda) B(\lambda), \quad b(\lambda)=\alpha^{*}(\lambda) B(\lambda)+\beta^{*}(\lambda) A(\lambda) .
$$


The function $a(\lambda)$ has the analytic continuation $a(z)$ for $z \in \mathbb{C}_{+}$, and $\bar{a}(z)=$ $a^{*}\left(z^{*}\right)$ is analytic in $\mathbb{C}_{-}$. The functions $b(\lambda)$ and $\bar{b}(\lambda)=b^{*}(\lambda)$ are defined for $\lambda \in \mathbb{R}$ only. The determinant of $T(\lambda) \equiv 1$ for $\operatorname{Im} \lambda=0$ and, hence, $|a(\lambda)|^{2}+|b(\lambda)|^{2} \equiv 1$. The spectral functions have the following asymptotics: $a(z)=1+O\left(z^{-1}\right)$ as $z \rightarrow \infty$ and $\operatorname{Im} z \geq 0$, and $b(\lambda)=O\left(\lambda^{-1}\right)$ as $\lambda \rightarrow \infty$.

If the function $a(z)$ has zeroes $z_{j} \in \mathbb{C}_{+}$, then

$$
a\left(z_{j}\right)=\operatorname{det}\left(Z[1]\left(t, x, z_{j}\right), Y[2]\left(t, x, z_{j}\right)\right)=0, \quad j=1,2, \ldots, p .
$$

Hence the vector columns of the determinant are linearly dependent:

$$
Y[2]\left(t, x, z_{j}\right)=\gamma_{j} Z[1]\left(t, x, z_{j}\right), \quad \gamma_{j}=\frac{B\left(z_{j}\right)}{\alpha\left(z_{j}\right)}=\frac{A\left(z_{j}\right)}{\beta\left(z_{j}\right)}, \quad j=1,2, \ldots, p .
$$

At the conjugated points $z_{j}^{*} \in \mathbb{C}_{-}(j=1,2, \ldots, p)$, the function

$$
\bar{a}\left(z_{j}^{*}\right)=\operatorname{det}\left(Y[1]\left(t, x, z_{j}^{*}\right), Z[2]\left(t, x, z_{j}^{*}\right)\right)=0 .
$$

Therefore,

$$
Y[1]\left(t, x, z_{j}^{*}\right)=\bar{\gamma}_{j} Z[2]\left(t, x, z_{j}^{*}\right), \quad \bar{\gamma}_{j}=\frac{B^{*}\left(z_{j}^{*}\right)}{\alpha^{*}\left(z_{j}^{*}\right)}=\frac{A^{*}\left(z_{j}^{*}\right)}{\beta^{*}\left(z_{j}^{*}\right)}=\gamma_{j}^{*} .
$$

Let us define the matrix

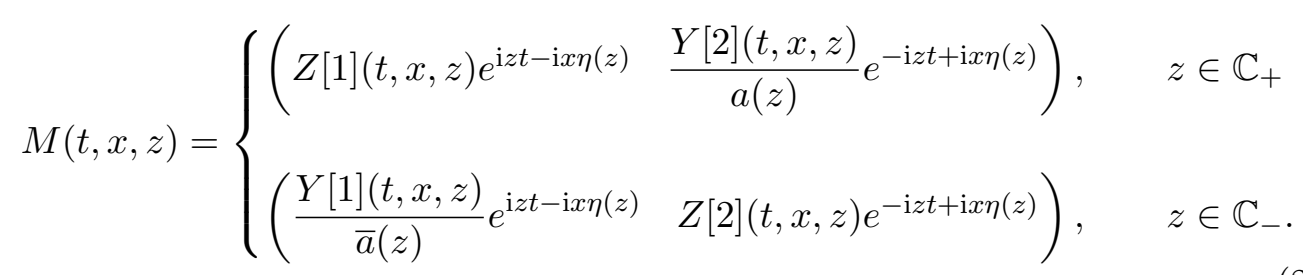

The matrix is analytic for $z \in \mathbb{C} \backslash \mathbb{R}$ if $a(z) \neq 0$, it has a jump across the real $\lambda$-axis $M(t, x, \lambda-\mathrm{i} 0)=M(t, x, \lambda+\mathrm{i} 0) J(t, x, \lambda)$, where

$$
J(t, x, \lambda)=\left(\begin{array}{cc}
1+|r(\lambda)|^{2} & -r(\lambda) e^{-2 \mathrm{i} \theta(t, x, \lambda)} \\
-\bar{r}(\lambda) e^{2 \mathrm{i} \theta(t, x, \lambda)} & 1
\end{array}\right), \quad \theta(t, x, \lambda)=\mathrm{i} z t-\mathrm{i} \eta(z) x,
$$

and $\operatorname{det} M(t, x, \lambda-\mathrm{i} 0)=\operatorname{det} M(t, x, \lambda+\mathrm{i} 0)=1$. The matrix $M(t, x, z)$ has the asymptotics $M(t, x, z)=I+O\left(z^{-1}\right)$ as $z \rightarrow \infty$. If $a(z)$ has zeroes, then the matrix is a meromorphic function and residues relations must be added. Namely, if the number of zeroes is finite and they are simple, i.e., $a\left(z_{j}\right)=0$ and $\dot{a}\left(z_{j}\right)=d a(z) /\left.d z\right|_{z=z_{j}} \neq 0(j=1,2, \ldots, p)$, then

$$
\underset{z=z_{j}}{\operatorname{res}} M[2](t, x, z)=m_{j} e^{-2 \mathrm{i} z_{j} t+2 \mathrm{i} x \eta\left(z_{j}\right)} M[1]\left(t, x, z_{j}\right),
$$




$$
\underset{z=z_{j}^{*}}{\operatorname{res}} M[1](t, x, z)=m_{j}^{*} e^{2 \mathrm{i} z_{j}^{*} t-2 \mathrm{i} x \eta\left(z_{j}^{*}\right)} M[2]\left(t, x, z_{j}^{*}\right),
$$

where $m_{j}=\gamma_{j} / \dot{a}\left(z_{j}\right), m_{j}^{*}=\bar{\gamma}_{j} / \dot{\bar{a}}^{-}\left(z_{j}^{*}\right)$, and the numbers $\gamma_{j}, \bar{\gamma}_{j}=\gamma_{j}^{*}$ are defined in (19) and (20).

\subsection{Mixed problem}

We consider here the mixed problem (6)-(7) in the domain $t \in \mathbb{R}_{+}, 0 \leq$ $x \leq L \leq \infty$. Let $\mathcal{E}(t, x), \mathcal{N}(t, x), \rho(t, x)$ be a smooth solution of the mixed problem. We use the compatible solution $Y(t, x, \lambda)$ ( with the restriction $t \in \mathbb{R}_{+}$) of the AKNS system of equations introduced in the previous subsection. Another compatible solution $\hat{Z}(t, x, \lambda)$ of equations (8)-(9) is defined as follows:

$$
\hat{Z}(t, x, \lambda)=\hat{\Psi}(t, x, \lambda) w(x, \lambda)
$$

where $\hat{\Psi}(t, x, \lambda)$ satisfies the $t$-equation for all $x$ and $\hat{\Psi}(0, x, \lambda)=I$, and $w(x, \lambda)$ satisfies the $x$-equation with $t=0$ under the initial condition $w(L, \lambda)=e^{\mathrm{i} L \eta(\lambda) \sigma_{3}}$ or $\lim _{x \rightarrow \infty} w(x, \lambda) e^{-\mathrm{i} x \eta(\lambda) \sigma_{3}}=I$ if $L=\infty$.

Lemma 2.4. Let $\mathcal{E}(t, x), \mathcal{N}(t, x), \rho(t, x)$ be smooth. The function $\hat{\Psi}(t, x, \lambda)$ has an integral representation

$$
\hat{\Psi}(t, x, \lambda)=e^{-\mathrm{i} \lambda t \sigma_{3}}+\int_{-t}^{t} L(t, \tau, x) e^{-\mathrm{i} \lambda \tau \sigma_{3}} \mathrm{~d} \tau .
$$

The kernel $L(t, \tau, x)$ is smooth, it satisfies the symmetry condition $L^{*}(t, \tau, x)=$ $\Lambda L(t, \tau, x) \Lambda^{-1}$ with the matrix $\Lambda=\left(\begin{array}{cc}0 & 1 \\ -1 & 0\end{array}\right)$, and

$$
\left[\sigma_{3}, L(t, t, x)\right]=H(t, x) \sigma_{3} .
$$

The proof of this lemma can be found in (cf. [17]). Integral representation (26) gives the analyticity of the Jost solution $\hat{\Psi}(t, x, z)$ for $z \in \mathbb{C}$ and its asymptotic (as $z \rightarrow \infty$ ) behavior:

$$
\begin{gathered}
\hat{\Psi}(t, x, z) e^{\mathrm{i} z t}=\left(\begin{array}{cc}
1 & 0 \\
0 & e^{2 \mathrm{i} z t}
\end{array}\right)+\mathrm{O}\left(z^{-1}\right)+\mathrm{O}\left(e^{2 \mathrm{i} z t} z^{-1}\right), \\
\hat{\Psi}(t, x, z) e^{-\mathrm{i} z t}=\left(\begin{array}{cc}
e^{-2 \mathrm{i} z t} & 0 \\
0 & 1
\end{array}\right)+\mathrm{O}\left(z^{-1}\right)+\mathrm{O}\left(e^{-2 \mathrm{i} z t} z^{-1}\right),
\end{gathered}
$$

where the symbol $\mathrm{O}($.$) means a matrix whose entries have the indicated order.$ 
The function $\hat{w}(x, \lambda)=w(x, \lambda) e^{-\mathrm{i} \eta(\lambda) x \sigma_{3}}$ must satisfy the integral equation

$$
\hat{w}(x, \lambda)=I+\int_{x}^{L} e^{\mathrm{i}(x-y) \eta(\lambda) \sigma_{3}} \hat{H}(y, \lambda) \hat{w}(y, \lambda) e^{-\mathrm{i}(x-y) \eta(\lambda) \sigma_{3}} \mathrm{~d} y,
$$

where $\hat{H}(y, \lambda)=H(0, y)+\mathrm{i}\left(F(0, y)+\sigma_{3}\right) / 4 \lambda$. By using this integral equation, we can prove that the matrix $w(x, \lambda)$ is analytic everywhere and has the asymptotic behavior

$$
w(x, \lambda)=\left(\begin{array}{cc}
e^{\mathrm{i} x \eta(\lambda)} & 0 \\
0 & e^{-\mathrm{i} x \eta(\lambda)}
\end{array}\right)+\left(\begin{array}{cc}
\chi_{11}(x, \lambda) e^{\mathrm{i} x \eta(\lambda)} & \chi_{12}(x, \lambda) e^{-\mathrm{i} x \eta(\lambda)} \\
\chi_{21}(x, \lambda) e^{\mathrm{i} x \eta(\lambda)} & \chi_{22}(x, \lambda) e^{-\mathrm{i} x \eta(\lambda)}
\end{array}\right)
$$

where $\chi_{11}(x, \lambda), \chi_{21}(x, \lambda)=\mathrm{O}\left(\lambda^{-1}\right)+\mathrm{O}\left(\lambda^{-1} e^{2 \mathrm{i} L \lambda}\right)$ and $\chi_{12}(x, \lambda), \chi_{22}(x, \lambda)=$ $\mathrm{O}\left(\lambda^{-1}\right)+\mathrm{O}\left(\lambda^{-1} e^{-2 \mathrm{i} L \lambda}\right)$ as $\lambda \rightarrow \pm \infty(0 \leq x \leq L<\infty)$. If $L=\infty$, then $\chi_{i j}(x, \lambda)=$ $\mathrm{O}\left(\lambda^{-1}\right)$ as $\lambda \rightarrow \pm \infty$. Due to the analytic continuation of the first vector column to the upper half-plane and the second vector column to the lower half-plane, we have $\left(\chi_{11}(x, z), \chi_{21}(x, z)\right)=\mathrm{O}\left(z^{-1}\right)$ for $\operatorname{Im} z \geq 0$ and $\left(\chi_{21}(x, z), \chi_{22}(x, z)\right)=$ $\mathrm{O}\left(z^{-1}\right)$ for $\operatorname{Im} z \leq 0$ as $z \rightarrow \infty$.

Formulas (25), (26), (27) and Lemmas 2.2, 2.4 imply the following properties of the matrices $\hat{Z}(t, x, \lambda)=(\hat{Z}[1](t, x, \lambda) \quad \hat{Z}[2](t, x, \lambda))$ :

1) $\hat{Z}(t, x, \lambda)(\lambda \neq 0)$ satisfies the $t$ - and $x$-equations $(8)-(9)$;

2) $\hat{Z}(t, x, \lambda)=\Lambda \hat{Z}^{*}\left(t, x, \lambda^{*}\right) \Lambda^{-1}, \lambda \in \mathbb{C} \backslash\{0\}$, where $\Lambda=\left(\begin{array}{cc}0 & 1 \\ -1 & 0\end{array}\right)$;

3) $\operatorname{det} \hat{Z}(t, x, \lambda) \equiv 1, \quad \lambda \in \mathbb{C} \backslash\{0\}$;

4) the map $(x, t) \longmapsto \hat{Z}(t, x, \lambda)(\lambda \neq 0)$ is smooth in $t$ and $x$;

5) the map $z \longmapsto \hat{Z}[1](t, x, z)$ is analytic in $z \in \mathbb{C}_{+}$;

6) the map $z \longmapsto \hat{Z}[2](t, x, z)$ is analytic in $z \in \mathbb{C}_{-}$;

7) the vector functions $\hat{Z}[1](t, x, \lambda) e^{\mathrm{i} \lambda t-\mathrm{i} x \eta(\lambda)}, \hat{Z}[2](t, x, z) e^{-\mathrm{i} \lambda t+\mathrm{i} x \eta(\lambda)}$ are analytic, bounded in $z \in \mathbb{C}_{ \pm}$, continuous up to the boundary $(\mathbb{R})$, and

$$
\begin{array}{cl}
\hat{Z}[1](t, x, z) e^{\mathrm{i} z t-\mathrm{i} x \eta(z)}=\left(\begin{array}{l}
1 \\
0
\end{array}\right)+O\left(z^{-1}\right), & z \in \mathbb{C}_{+}, \quad z \rightarrow \infty ; \\
\hat{Z}[2](t, x, z) e^{-\mathrm{i} z t+\mathrm{i} x \eta(z)}=\left(\begin{array}{l}
0 \\
1
\end{array}\right)+O\left(z^{-1}\right), & z \in \mathbb{C}_{-}, \quad z \rightarrow \infty .
\end{array}
$$

Since the matrices $Y(t, x, \lambda), \hat{Z}(t, x, \lambda)$ are the solutions of Eqs. (8)-(9), they are linearly dependent. Thus, there exists a transition matrix $T(\lambda)$, independent of $x$ and $t$, such that

$$
Y(t, x, \lambda)=\hat{Z}(t, x, \lambda) T(\lambda) .
$$

The transition matrix is equal to

$$
T(\lambda)=\hat{Z}^{-1}(0,0, \lambda) Y(0,0, \lambda)=w^{-1}(0, \lambda) \Phi(0, \lambda)
$$


and, hence, $T(\lambda)=\Lambda T^{*}(\lambda) \Lambda^{-1}$, i.e., $T(\lambda)$ has the form

$$
T(\lambda)=\left(\begin{array}{cc}
\bar{a}(\lambda) & b(\lambda) \\
-\bar{b}(\lambda) & a(\lambda)
\end{array}\right)
$$

For the convenience of further formulation of the $\mathrm{RH}$ problem, we use the same notations as in the previous subsection for the transition matrix $T(\lambda)$ and its entries. It is easy to see that the matrix $w(0, \lambda)=\hat{w}(0, \lambda)=\left(\begin{array}{cc}\alpha(\lambda) & -\bar{\beta}(\lambda) \\ \beta(\lambda) & \bar{\alpha}(\lambda)\end{array}\right)$ is the spectral function of the $x$-equation for $t=0$. It is uniquely defined by the given initial functions $\mathcal{E}(0, x), \rho(0, x)$ and $\mathcal{N}(0, x)$. The function $\Phi(0, \lambda)$ is the spectral function of the $t$-equation for $x=0$, uniquely defined by the boundary condition $\mathcal{E}(t, 0)$, and it has the form $\Phi(0, \lambda)=\left(\begin{array}{cc}\bar{A}(\lambda) & B(\lambda) \\ -\bar{B}(\lambda) & A(\lambda)\end{array}\right)$. The functions $\alpha(\lambda), \beta(\lambda)$ and $A(\lambda), B(\lambda)$ have analytic continuations in the upper half-plane $\mathbb{C}_{+}$, and $\bar{\alpha}(\lambda)=\alpha^{*}(\lambda), \bar{\beta}(\lambda)=\beta^{*}(\lambda)$ and $\bar{A}(\lambda)=A^{*}(\lambda), \bar{B}(\lambda)=B^{*}(\lambda)$ have analytic continuations in the lower half-plane $\mathbb{C}_{-}$. Thus we have

$$
\begin{gathered}
a(z)=\alpha(z) A(z)-\beta(z) B(z), \quad z \in \mathbb{C}_{+} \cup \mathbb{R} ; \\
b(\lambda)=\alpha^{*}(\lambda) B(\lambda)+\beta^{*}(\lambda) A(\lambda), \quad \lambda \in \mathbb{R} .
\end{gathered}
$$

The function $a(z)$ is analytic in $\mathbb{C}_{+}$, and $\bar{a}(z)=a^{*}\left(z^{*}\right)$ is analytic in $\mathbb{C}_{-}$. The functions $b(\lambda)$ and $\bar{b}(\lambda)=b^{*}(\lambda)$ are defined for $\lambda \in \mathbb{R}$ only. The determinant of $T(\lambda) \equiv 1$ for $\operatorname{Im} \lambda=0$ and, hence $|a(\lambda)|^{2}+|b(\lambda)|^{2} \equiv 1$. The spectral functions have the following asymptotics:

$$
a(z)=1+O\left(z^{-1}\right), \quad z \rightarrow \infty, \quad b(\lambda)=O\left(\lambda^{-1}\right), \quad \lambda \rightarrow \infty .
$$

If $a(z)(\bar{a}(z))$ has zeroes, then we have the relation between the vector columns $\hat{Z}[1]\left(t, x, z_{j}\right)$ and $Y[2]\left(t, x, z_{j}\right)\left(Y[1]\left(t, x, z_{j}^{*}\right)\right.$ and $\left.\hat{Z}[2]\left(t, x, z_{j}^{*}\right)\right)(j=1,2, \ldots, p)$ similarly to (19), (20).

Further, the matrix

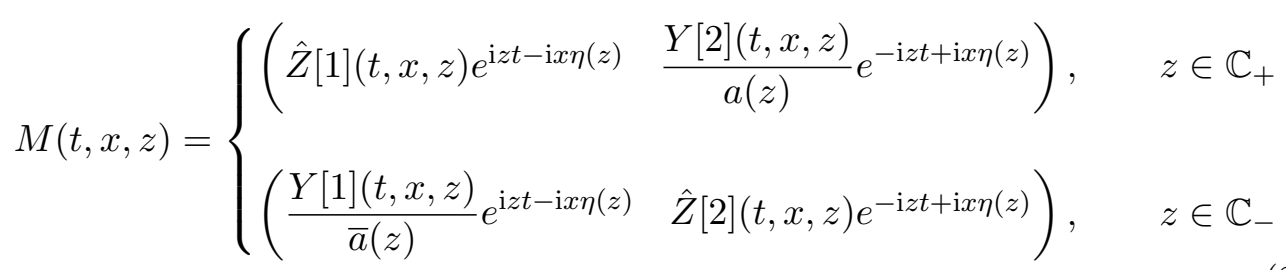

and the scattering relation (28) generate explicitly the same matrix $\mathrm{RH}$ problem as in (21), (22), (23), (24). 


\section{Matrix Riemann-Hilbert Problems}

In this section we give a reconstruction of the solution to the MB equations in terms of the spectral functions $a(\lambda), b(\lambda)$, which are defined through the spectral functions $A(\lambda), B(\lambda)$ and $\alpha(\lambda), \beta(\lambda)$.

In the previous section we have proved that the matrices (21), (29) (due to the scattering relations (18), (28)) are the solutions of the following matrix $\mathrm{RH}$ problem $R H_{t x}$ :

Find the $2 \times 2$ matrix $M(t, x, z)$ such that

- $M(t, x, z)$ is analytic ( if $a(z) \neq 0$ ) or meromorphic (if $a\left(z_{j}\right)=\bar{a}\left(z_{j}^{*}\right)=$ $\left.0, \operatorname{Im} z_{j}>0, j=1,2, \ldots, p\right)$ in $z \in \mathbb{C} \backslash \mathbb{R}$ and continuous up to the real $\lambda$-axis;

RH 1

- If $a\left(z_{j}\right)=\bar{a}\left(z_{j}^{*}\right)=0, j=1,2, \ldots, p$, then $M(x, t, z)$ has poles at the points $z=z_{j}, z=z_{j}^{*}(j=1,2, \ldots, p)$, and the corresponding residues satisfy the relations:

$$
\begin{aligned}
& \underset{z=z_{j}}{\operatorname{res}} M[2](t, x, z)=m_{j} e^{-2 \mathrm{i} z_{j} t+2 \mathrm{i} x \eta\left(z_{j}\right)} M[1]\left(t, x, z_{j}\right) \\
& \underset{z=z_{j}^{*}}{\operatorname{res}} M[1](t, x, z)=m_{j}^{*} e^{2 \mathrm{i} z_{j}^{*} t-2 \mathrm{i} x \eta\left(z_{j}^{*}\right)} M[2]\left(t, x, z_{j}^{*}\right),
\end{aligned}
$$

where $m_{j}=\gamma_{j} / \dot{a}\left(z_{j}\right), m_{j}^{*}=\bar{\gamma}_{j} / \dot{\bar{a}}\left(z_{j}^{*}\right)$, and the numbers $\gamma_{j}, \bar{\gamma}_{j}=\gamma_{j}^{*}$ are defined in (19) and (20);

- $M_{-}(t, x, \lambda)=M_{+}(t, x, \lambda) J(t, x, \lambda), \quad \lambda \in \mathbb{R}$,

RH4

$$
J(t, x, \lambda)=\left(\begin{array}{cc}
1+|r(\lambda)|^{2} & -r(\lambda) e^{-2 \mathrm{i} \theta(t, x, \lambda)} \\
-\bar{r}(\lambda) e^{2 \mathrm{i} \theta(t, x, \lambda)} & 1
\end{array}\right), \quad \lambda \in \mathbb{R}
$$

where $r(\lambda)=b(\lambda) / a(\lambda)$ and $\theta(t, x, \lambda)=\lambda t-x \eta(\lambda)$.

- $M(t, x, z)=I+O\left(z^{-1}\right), \quad|z| \rightarrow \infty$.

RH5

Taking into account the well-known fact that $a(z)$ can have multiple zeros or infinitely many zeros with limit points on the real $\lambda$ - axis or real zeroes (the socalled spectral singularities), we propose below a more convenient formulation of the matrix $\mathrm{RH}$ problem. We introduce once more the matrix solution $Z_{0}(t, x, \lambda)$ of the AKNS system of equations normalized by the condition $Z_{0}(0,0, \lambda)=I$. It is seen that $Z_{0}(t, x, \lambda)=\hat{\Psi}(t, x, \lambda) W(0, x, \lambda)$, where $\hat{\Psi}(t, x, \lambda)$ and $W(t, x, \lambda)$ were defined in the first section. Thus, $Z_{0}(t, x, \lambda)$ is analytic in $z \in \mathbb{C} \backslash\{0\}$, $Z_{0}(t, x, \lambda) e^{\mathrm{i} \theta(t, x, \lambda) \sigma_{3}}$ is bounded in any disk $|z| \leq R$. Hence the matrix can be 
extended up to the continuous function in the disk. Then we put

$$
\hat{M}(t, x, z)=\left\{\begin{array}{lc}
M(t, x, z), & |z|>R \\
Z_{0}(t, x, \lambda) e^{\mathrm{i} \theta(t, x, \lambda) \sigma_{3}}, & |z|<R
\end{array}\right.
$$

where $R$ is positive and sufficiently large such that $a(z) \neq 0$ when $|z|>R$. Let the contour $\Sigma_{R}=(-\infty,-R] \cup[R, \infty) \cup \Gamma_{R}$, where $\Gamma_{R}=\{z:|z|=R\}$ is the circle of radius $R$ oriented clockwise. Then we obtain the equivalent $\mathrm{RH}$ problem:

- $\hat{M}(t, x, z)$ is analytic in $z \in \mathbb{C} \backslash \Sigma_{R}$ and continuous up to the contour $\Sigma_{R}$; $R R H 1$

- $\hat{M}_{-}(t, x, z)=\hat{M}_{+}(t, x, z) J(t, x, z), \quad z \in \Sigma_{R}$,

RRH2

$$
J(t, x, z)=\left\{\begin{array}{l}
\left(\begin{array}{cc}
1+|r(\lambda)|^{2} & -r(\lambda) e^{-2 \mathrm{i} \theta(t, x, \lambda)} \\
-\bar{r}(\lambda) e^{2 \mathrm{i} \theta(t, x, \lambda)} & 1
\end{array}\right), \quad z=\lambda \in \mathbb{R} \backslash(-R, R), \\
\left(\begin{array}{cc}
\alpha(z) & B(z) e^{-2 \mathrm{i} \theta(t, x, z)} / a(z) \\
\beta(z) e^{2 \mathrm{i} \theta(t, x, z)} & A(z) / a(z)
\end{array}\right), \quad z \in \Gamma_{R} \cap \mathbb{C}_{+}, \\
\left(\begin{array}{cc}
\bar{A}(z) / \bar{a}(z) & -\bar{\beta}(z) e^{-2 \mathrm{i} \theta(t, x, z)} \\
-\bar{B}(z) e^{2 \mathrm{i} \theta(t, x, z) / \bar{a}(z)} & \bar{\alpha}(z)
\end{array}\right), \quad z \in \Gamma_{R} \cap \mathbb{C}_{-} .
\end{array}\right.
$$

- $\hat{M}(t, x, z)=I+O\left(z^{-1}\right), \quad|z| \rightarrow \infty$.

RRH3

We will prove now the following theorem.

Theorem 3.1. Let the functions $\mathcal{E}(t, x), \mathcal{N}(t, x)$ and $\rho(t, x)$ be the solutions to the Maxwell-Bloch equations (1)-(3) considered in the subsection 2.1 or 2.2 . There exists the matrix $M(t, x, z)$ which is the solution of the Riemann-Hilbert problem (RRH1)-(RRH3), and the complex electric field envelope $\mathcal{E}(t, x)$ is defined by the relation

$$
\mathcal{E}(t, x)=-\lim _{z \rightarrow \infty} 4 \mathrm{i} z M_{12}(t, x, z) .
$$

The entries $\mathcal{N}(t, x)$ and $\rho(t, x)$ of the matrix $F(t, x)$ are defined as follows:

$$
F(t, x)=-m_{0}(t, x) \sigma_{3} m_{0}^{-1}(t, x), \quad m_{0}(t, x)=\lim _{z \rightarrow 0} M(t, x, z) .
$$


P r o o f. The only thing we need is to prove Eq. (32). The matrix $M(t, x, z)$ defines the solution $\Phi(t, x, z)$ of the AKNS Eqs. (8) and (9) by the formula

$$
\Phi(t, x, z)=M(t, x, z) e^{-\mathrm{i} \theta(t, x, z) \sigma_{3}} .
$$

Formulas (32) follow from (8) and (RRH3). Indeed, substituting the last formula into equation (8), we can find that

$$
M_{t}+\mathrm{i} z\left[\sigma_{3}, M\right]+H M=0 .
$$

Using (RRH3), we put

$$
M(t, x, z)=I+\frac{m(t, x)}{z}+\mathrm{o}\left(z^{-1}\right)
$$

where

$$
m(t, x)=\lim _{z \rightarrow \infty} z(M(t, x, z)-I) .
$$

This asymptotics and Eq. (34) give

$$
H(t, x)=-\mathrm{i}\left[\sigma_{3}, m(t, x)\right],
$$

and hence

$$
\mathcal{E}(t, x)=-4 \mathrm{i} m_{12}=-\lim _{z \rightarrow \infty} 4 \mathrm{i} z M_{12}(t, x, z) .
$$

Further, since $M(t, x, z)=m_{0}(t, x)+\mathrm{O}\left(z^{-1}\right)$, then the $x$-equation for $M(t, x, z)$,

$$
M_{x}-\frac{\mathrm{i}}{4 z} M \sigma_{3}=\mathrm{i} z\left[\sigma_{3}, M\right]+H M+\frac{\mathrm{i} F}{4 z} M
$$

gives $F(t, x)=-m_{0}(t, x) \sigma_{3} m_{0}^{-1}(t, x)$.

Thus the problem to the Maxwell-Bloch equations, from subsection 2.1 on the whole t-line and the mixed problem from subsection 2.2 in the quarter $x$-plane, is completely linearizable.

\section{More General Matrix Riemann-Hilbert Problems}

Now we prove that any Riemann-Hilbert problem like $R R H 1-R R H 3$ generates a solution to the Maxwell-Bloch equations. From here and below we will consider a more general construction. Let the oriented contour $\Sigma$ contain a real line $\mathbb{R}$, sufficiently large circle $\Gamma$ and some finite $\operatorname{arcs} \gamma_{j} \cup \bar{\gamma}_{j}(j=1,2, \ldots, p)$ which are symmetric with respect to the real line. Thus,

$$
\Sigma=\mathbb{R} \cup \Gamma \cup \bigcup_{j=1}^{p} \gamma_{j} \cup \bar{\gamma}_{j} .
$$


This type of contours takes place when we deal with periodic initial data or/and periodic boundary conditions. The contour $\Sigma$ has the following orientation: the real line $\mathbb{R}$ is oriented from the left to the right, the circle $\Gamma$ is oriented clockwise, the $\operatorname{arcs} \gamma_{j} \cup \bar{\gamma}_{j}$ are oriented up-down. Then the regular matrix $\mathrm{RH}$ problem can be formulated as follows.

Find the $2 \times 2$ matrix $M(t, x, z)$ such that

- $M(t, x, z)$ is analytic in $z \in \mathbb{C} \backslash \Sigma$ and bounded up to the contour $\Sigma ; \quad R 1$

- $M_{-}(t, x, z)=M_{+}(t, x, z) J(t, x, z), \quad z \in \Sigma, \quad R 2$

- $M(t, x, z)=I+O\left(z^{-1}\right), \quad|z| \rightarrow \infty$.

The contour $\Sigma$ and the jump matrix $J(x, t, z)$ satisfy the Schwartz reflection principle:

- the contour $\Sigma$ is symmetric with respect to the real axis $\mathbb{R}$,

- $J^{-1}(x, t, z)=J^{\dagger}\left(x, t, z^{*}\right)$ for $z \in \Sigma$ and $\operatorname{Im} z \neq 0$,

where $\dagger$ and $*$ are Hermitian and complex conjugations, respectively.

- the jump matrix $J(x, t, \lambda)$ for $\lambda \in \mathbb{R}$ has a positive definite real part.

Theorem 4.1. Let the jump matrix $J(t, x, z)$ satisfy the Schwartz reflection principle and $I-J(t, x,.) \in L^{2}(\Sigma) \cap L^{\infty}(\Sigma)$. Then for any fixed $t, x \in \mathbb{R}$, the regular $R H$ problem $R 1, R 2, R 3$ has a unique solution $M(t, x, z)$.

$\mathrm{P}$ r o o f. Existence. Let $x$ and $t$ be fixed. We are to find the solution $M(t, x, z)$ of the $\mathrm{RH}$ problem in the form

$$
M(t, x, z)=I+\frac{1}{2 \pi \mathrm{i}} \int_{\Sigma} \frac{P(t, x, s)[I-J(t, x, s)]}{s-z} d s, \quad z \notin \Sigma .
$$

The Cauchy integral (36) provides all properties of the $\mathrm{RH}$ problem (cf.[22]) if and only if the matrix $Q(t, x, \lambda):=P(t, x, \lambda)-I$ satisfies the singular integral equation

$$
Q(t, x, z)-\mathcal{K}[Q](t, x, z)=R(t, x, z), \quad z \in \Sigma .
$$

The singular integral operator $\mathcal{K}$ and the right-hand side $R(t, x, z)$ are as follows:

$$
\begin{aligned}
\mathcal{K}[Q](t, x, z) & :=\frac{1}{2 \pi \mathrm{i}} \int_{\Sigma} \frac{Q(t, x, s)[I-J(t, x, s)]}{s-z_{+}} d s, \\
R(t, x, z) & :=\quad \frac{1}{2 \pi \mathrm{i}} \int_{\Sigma} \frac{I-J(t, x, s)}{s-z_{+}} d s .
\end{aligned}
$$


We consider this integral equation in the space $L^{2}(\Sigma)$ of the $2 \times 2$ matrix complex valued functions $Q(z):=Q(t, x, z), z \in \Sigma$. The norm of $Q \in L^{2}(\Sigma)$ is given by

$$
\left.\|Q\|_{L^{2}(\Sigma)}=\left(\int_{\Sigma} \operatorname{tr}\left(\mathrm{Q}^{\dagger}(\mathrm{z}) \mathrm{Q}(\mathrm{z})\right)|\mathrm{dz}|\right)^{1 / 2}=\left.\left(\sum_{j, l=1}^{2} \int_{\Sigma} \mid Q_{j l}(z)\right)\right|^{2}|d z|\right)^{1 / 2} .
$$

The operator $\mathcal{K}$ is defined by the jump matrix $J(t, x, z)$ and the generalized function

$$
\frac{1}{s-z_{+}}=\lim _{z^{\prime} \rightarrow z, z^{\prime} \in \text { side }+} \frac{1}{s-z^{\prime}} .
$$

Furthermore, since the jump matrix $J(t, x, \lambda)$ has a positive definite real part when $\lambda \in \mathbb{R}$, then Theorem 9.3 from [23] (p. 984) guarantees the $L^{2}$ invertibility of the operator $I d-\mathcal{K}$ ( $I d$ is the identical operator). The function $R(t, x, z)$ belongs to $L^{2}(\Sigma)$ because $\left.I-J(t, x, z) \in L^{2} \Sigma\right)$ when $z \in \Sigma$, and the Cauchy operator

$$
C_{+}[f](z):=\frac{1}{2 \pi \mathrm{i}} \int_{\Sigma} \frac{f(s)}{s-z_{+}} d s=\frac{f(z)}{2}+\text { p.v. } \frac{1}{2 \pi \mathrm{i}} \int_{\Sigma} \frac{f(s)}{s-z} d s
$$

is bounded in the space $L^{2}(\Sigma)$ [24]. Therefore, the singular integral Eq. (37) has a unique solution $Q(t, x, z) \in L^{2}(\Sigma)$ for any fixed $x, t \in \mathbb{R}$, and formula (36) gives the solution of the above $\mathrm{RH}$ problem.

Uniqueness. The proof is as follows. Since $\operatorname{det} J(t, x, z) \equiv 1$, one can find that $\operatorname{det} M(t, x, z) \equiv 1$ by repeating step by step the proof of Theorem 7.18 from [22] (p. 194-198). Hence the matrix $M^{-1}(t, x, z)$ exists and it is analytic in $z \in \mathbb{C} \backslash \Sigma$. Let us now suppose that there is another matrix $\tilde{M}(t, x, z)$ which solves the given Riemann-Hilbert problem. Thus,

$$
\begin{gathered}
\tilde{M}_{-}(t, x, z) M_{-}^{-1}(t, x, z)=\tilde{M}_{+}(t, x, z) J(t, x, z) J^{-1}(t, x, z) M_{+}^{-1}(t, x, z) \\
=\tilde{M}_{+}(t, x, z) M_{+}^{-1}(t, x, z),
\end{gathered}
$$

and we can find that the matrix $\tilde{M}(t, x, z) M^{-1}(t, x, z)$ is analytic in $z \in \mathbb{C}$ and it tends to the identity matrix as $z \rightarrow \infty$. By Liovilles's theorem, $\tilde{M}(t, x, z) M^{-1}(t, x, z) \equiv I$ and therefore $\tilde{M}(t, x, z) \equiv M(t, x, z)$, i.e., the matrix $M(t, x, z)$ is unique.

Theorem 4.2. Let $M(t, x, z)$ be the solution of the $R H$ problem $(R 1)-(R 3)$ given by Theorem 4.1 with a matrix $J(t, x, z)$ such that

$$
J(t, x, z)=e^{(-\mathrm{i} z t+\mathrm{i} \eta(z) x) \sigma_{3}} J_{0}(z) e^{(\mathrm{i} z t-\mathrm{i} \eta(z) x) \sigma_{3}}, \quad \eta(z)=z-\frac{1}{4 z},
$$


where $J_{0}(z)$ is independent of $t$ and $x$. If additionally $M(t, x, z)$ is smooth in $t$ and $x$, then the matrix $\Phi(t, x, z):=M(t, x, z) e^{(-\mathrm{i} z t+\mathrm{i} \eta(z) x) \sigma_{3}}$ satisfies the AKNS system of Eqs. (8) and (9) with the functions $\mathcal{E}(t, x), \mathcal{N}(t, x), \rho(t, x)$ given by (32) and (33). Moreover, they are smooth and satisfy the MB Eqs. (1)-(3).

P r o o f. The matrix $\Phi(t, x, z):=M(t, x, z) e^{(-\mathrm{i} z t+\mathrm{i} \eta(z) x) \sigma_{3}}$ is analytic in $z \in \mathbb{C} \backslash \Sigma$ and has the jump across $\Sigma$ :

$$
\Phi_{-}(t, x, z)=\Phi_{+}(t, x, z) J_{0}(z),
$$

where $J_{0}(\lambda)$ is independent of $t$ and $x$. This relation implies:

$$
\begin{aligned}
& \frac{d \Phi_{-}(t, x, z)}{d t} \Phi_{-}^{-1}(t, x, z)=\frac{d \Phi_{+}(t, x, z)}{d t} \Phi_{+}^{-1}(t, x, z), \\
& \frac{d \Phi_{-}(t, x, z)}{d x} \Phi_{-}^{-1}(t, x, z)=\frac{d \Phi_{+}(t, x, z)}{d x} \Phi_{+}^{-1}(t, x, z)
\end{aligned}
$$

for $z \in \Sigma$. The last relations mean that the matrix logarithmic derivatives $\Phi_{t}(t, x, z) \Phi^{-1}(t, x, z)$ and $\Phi_{x}(t, x, z) \Phi^{-1}(t, x, z)$ are analytic in $z \in \mathbb{C} \backslash\{0\}$ except the end points and the points of self intersection of the contour $\Sigma$. The matrix $M(t, x, z)$ and its derivative $M_{t}(t, x, z)$ (in $t$ ) are analytic in $z \in \mathbb{C} \backslash \Sigma$. Moreover, the Cauchy integral (36) gives the following asymptotic formulas:

$$
M(t, x, z)=I+\frac{m_{ \pm}(t, x)}{z}+\mathrm{O}\left(z^{-2}\right), \quad z \rightarrow \infty, \quad z \in \mathbb{C}_{ \pm}
$$

Hence

$$
\begin{aligned}
\Phi_{t}(t, x, z) \Phi^{-1}(t, x, z) & =-\mathrm{i} z \sigma_{3}+\mathrm{i}\left[\sigma_{3}, m_{+}(t, x)\right]+\mathrm{O}\left(z^{-1}\right) \\
& =-\mathrm{i} z \sigma_{3}+\mathrm{i}\left[\sigma_{3}, m_{-}(t, x)\right]+\mathrm{O}\left(z^{-1}\right), \quad z \rightarrow \infty,
\end{aligned}
$$

where $[A, B]:=A B-B A$ and

$$
m_{-}(t, x)=m_{+}(t, x)=m(t, x)=\frac{\mathrm{i}}{2 \pi} \int_{\Sigma} P(t, x, z)[I-J(t, x, z)] d z .
$$

Since $M(t, x, z)$ is bounded up to the boundary, then $z=0$, and the end points and the points of self-intersection of the contour $\Sigma$ are removable singularities for $\Phi_{t}(t, x, z) \Phi^{-1}(t, x, z)$. Therefore, by Liouville's theorem, this derivative is a polynomial

$$
U(z):=\Phi_{t}(t, x, z) \Phi^{-1}(t, x, z)=-\mathrm{i} z \sigma_{3}-H(t, x),
$$

where $H(t, x):=-\mathrm{i}\left[\sigma_{3}, m(t, x)\right]=\left(\begin{array}{cc}0 & q(t, x) \\ p(t, x) & 0\end{array}\right)$. Using the Schwartz symmetry properties of the jump matrix $J(t, x, z)$, we can show that $U(z)=\sigma_{2} U^{*}\left(z^{*}\right) \sigma_{2}$, 
where $\sigma_{2}=\left(\begin{array}{cc}0 & -\mathrm{i} \\ \mathrm{i} & 0\end{array}\right)$. These reductions imply $H(t, x)=-H^{\dagger}(x, t)$, i.e., $q(t, x)=$ $-p^{*}(t, x)$, and we put $q(t, x):=\mathcal{E}(t, x) / 2$. Thus $\Phi(t, x, z)$ satisfies Eq. (8), and a scalar function $\mathcal{E}(t, x)$ is defined by $(32)$. The function $\mathcal{E}(t, x)$ is smooth in $t$ and $x$ because the matrix $M(x, t, z)$, and hence $m(t, x)$ are smooth in $t$ and $x$ by supposition. In the same way as before, we can find that $\Phi_{x}(x, t, \lambda) \Phi^{-1}(x, t, \lambda)$ is a rational matrix function

$$
V(z):=\Phi_{x}(x, t, \lambda) \Phi^{-1}(x, t, \lambda)=\mathrm{i} z \sigma_{3}+H(t, x)+\frac{\mathrm{i} \hat{F}(t, x)}{4 z}
$$

because the following asymptotics are true:

$$
\Phi_{x}(t, x, z) \Phi^{-1}(t, x, z)=\mathrm{i} z \sigma_{3}+H(t, x)+\mathrm{O}\left(z^{-1}\right), \quad z \rightarrow \infty,
$$

and

$$
\Phi_{x}(t, x, z) \Phi^{-1}(t, x, z)=-\frac{\mathrm{i} \hat{F}(t, x)}{4 z}+F_{0}(t, x)+\mathrm{O}(z), \quad z \rightarrow 0,
$$

where $\hat{F}(t, x)=-M(t, x, 0) \sigma_{3} M^{-1}(t, x, 0)$, and $F_{0}(t, x)$ is some matrix. Moreover, the previous relations give: $F_{0}(t, x) \equiv H(t, x)$. Thus the matrix $\Phi(x, t, z)$ satisfies two differential equations:

$$
\begin{aligned}
\Phi_{t} & =U(z) \Phi, & U(z) & =-\mathrm{i} z \sigma_{3}-H(t, x) \\
\Phi_{x} & =V(z) \Phi, & V(z) & =\mathrm{i} z \sigma_{3}+H(t, x)+\frac{\mathrm{i} \hat{F}(t, x)}{4 z} .
\end{aligned}
$$

Their compatibility $\left(\Phi_{x t}(x, t, \lambda)=\Phi_{t x}(x, t, \lambda)\right)$ gives the identity in $z$,

$$
U_{x}(z)-V_{t}(z)+[U(z), V(z)]=0, \quad[U, V]=U V-V U
$$

i.e.,

$$
H_{t}(t, x)+H_{x}(t, x)+\left[\mathrm{i} z \sigma_{3}+H(t, x), \mathrm{i} z \sigma_{3}+H(t, x)+\frac{\mathrm{i} \hat{F}(t, x)}{4 z}\right]=0 .
$$

This identity is equivalent to the system of matrix equations:

$$
\begin{aligned}
H_{t}(t, x)+H_{x}(t, x) & =\frac{1}{4}\left[\sigma_{3}, \hat{F}(t, x)\right] \\
\hat{F}_{t}(t, x) & =[\hat{F}(t, x), H(t, x)] .
\end{aligned}
$$

Using the Schwartz symmetry properties of the jump matrix $J(t, x, z)$, we find that $\hat{F}(t, x)$ is a Hermitian matrix, and we put

$$
\hat{F}(t, x)=\left(\begin{array}{cc}
\mathcal{N}(t, x) & \rho(t, x) \\
\rho^{*}(t, x) & -\mathcal{N}(t, x)
\end{array}\right)
$$


Matrix Eqs. (40) and (41) are equivalent to the scalar Eqs. (6). Thus we have proved that the matrix $\Phi(t, x, z)$ satisfies equations (38) and (39) that coincide with AKNS system (8) and (9), and the scalar functions $\mathcal{E}(t, x), \mathcal{N}(t, x), \rho(t, x)$ are smooth and satisfy the MB equations (6) due to the compatibility of equations (38) and (39).

As a corollary of the previous theorems, we obtain (due to formulas (32), (36)) an integral representation for the electric field envelope

$$
\mathcal{E}(t, x)=\frac{2}{\pi} \int_{\Sigma}([I+Q(t, x, z)][J(t, x, z)-I])_{12} d z
$$

through the solution $Q(t, x, z)$ of the singular integral equation (37) which is equivalent to the regular $\mathrm{RH}$ problem. The entries $\mathcal{N}(t, x)$ and $\rho(t, x)$ of the density matrix of a quantum two-level atom subsystem are defined by

$$
\left(\begin{array}{cc}
\mathcal{N}(t, x) & \rho(t, x) \\
\rho^{*}(t, x) & -\mathcal{N}(t, x)
\end{array}\right)=-M(t, x, 0) \sigma_{3} M^{-1}(t, x, 0)
$$

or by using linear differential equations (2) and (3) by already known $\mathcal{E}(t, x)$.

\section{Conclusions}

Thus the Riemann-Hilbert problem $R 1-R 3$ with the given contour conjugation and the jump matrix given by Theorem 4.2, which satisfy the Schwartz reflection principle, generates the solutions to the Maxwell-Bloch equations. Among them there are the solutions defined for $t \in \mathbb{R}, x \in \mathbb{R}_{+}$and studied in $[5,6]$, the step-like solutions with a different background shape as $t \rightarrow \pm \infty$ that (by the best of our knowledge) are not considered in the literature, the solutions to the mixed problem $(6),(7)\left(t, x \in \mathbb{R}_{+}\right)$with decreasing or periodic input pulse $\mathcal{E}(t, 0)$ and different initial functions $\mathcal{E}(0, x), \mathcal{N}(0, x), \rho(0, x)$, etc. The type of solutions is defined by the specialization of the conjugation contour and the jump matrix on this contour. The specialization which cover the periodicity case and description of the corresponding solutions will be done further.

Acknowledgments. This research was supported by the grant Network of Mathematical Research 2013-2015

\section{References}

[1] G.L. Lamb Jr., Propagation of Ultrashort Optical Pulses. - Phys. Lett. A 25A (1967), 181-182.

[2] G.L. Lamb Jr., Analytical Ddescriptions to Ultrashort Optical Pulse Propagation in Resonant Media. - Rev. Mod. Phys. 43 (1971), 99-124. 
[3] G.L. Lamb Jr., Phase Variation in Coherent-optical-pulse Propagation. - Phys. Rev. Lett. 31 (1973), 196-199.

[4] G.L. Lamb Jr., Coherent-Optical-Pulse Propagation as an Inverse Problem. Phys. Rev. A 9 (1974), 422-430.

[5] M.J. Ablowits, D. Kaup, and A.C. Newell, Coherent-Pulse Propagation, a Dispersive, Irreversible Phenomenon. — J. Math. Phys. 15 (1974), 1852-1858.

[6] I.R. Gabitov, V.E. Zakharov, and A.V. Mikhailov, Maxwell-Bloch Equations and Inverse Scattering Transform Method. - Teor. Mat. Fiz. 63 (1985), 11-31.

[7] P. Deift and X. Zhou, A Steepest Descent Method for Oscillatory Riemann-Hilbert Problems. Asymptotics for the MKdV Equation. - Ann. Math. 137/2 (1993), 295368.

[8] V.P. Kotlyarov and A.A. Minakov, Step-Initial Function to the MKdV Equation: Hyperelliptic Long-Time Asymptotics of the Solution. - J. Math. Phys., Anal., Geom. 8 (2012), No. 1, 38-62.

[9] M.J. Ablowitz and H. Segur, Solitons and the Inverse Scattering Transform. SIAM, Philadelphia, 1981.

[10] S. V. Manakov, Propagation of Ultrshort Optical Pulse in a Two-Level Laser Amplifier. - Zh. Eksp. Teor. Fiz. 83 (1982), 68-83.

[11] S.V. Manakov and V.Yu. Novokshenov, Complete Asymptotic Representation of Electromagnetic Pulse in a Long Two-Level Amplifier. - Teor. Mat. Fiz. 69 (1986), $40-54$.

[12] O.M. Kiselev, Solution of Goursat Problem for Maxwell-Bloch Equations. - Teor. Mat. Fiz. 98 (1994), 29-37.

[13] A.S. Fokas, A Unified Transform Method for Solving Linear and Certain Nonlinear PDEs. - Proc. R. Soc. London, Ser. A 453 (1997), 1411-1443.

[14] A.S. Fokas and A.R. Its, The Linearization of the Initial Boundary Value Problem of the Nonlinear Schrödinger Equation. — SIAM J. Math. Anal. 27 (1996), 738-764.

[15] A.S. Fokas and A.R. Its, An Initial Boundary Value Problem for the Korteweg de Vries Equation. - Mathematics and Computer in Simulation 37 (1994), 293-321.

[16] A.S. Fokas and A.R. Its, An Initial Boundary Value Problem for the sine-Gordon Equation in laboratory coordinates. - Teor. Mat. Fiz. 92 (1992), 387-403.

[17] A. Boutet de Monvel and V.P. Kotlyarov, Scattering Problem for the ZakharovShabat Equations on the Semi-Axis. - Inverse Probl. 16 (2000), 1813-1837.

[18] A. Boutet de Monvel and V.P. Kotlyarov, Generation of Asymptotic Solitons of the Nonlinear Schrödinger Equation by Boundary Data. — J. Math. Phys. 44 (2003), 3185-3215.

[19] A. Boutet de Monvel and V. Kotlyarov, Focusing Nonlinear Schrödinger Equation on the Quarter Plane with Time-Periodic Boundary Condition: a Riemann-Hilbert Approach. - Journal of the Institute of Mathematics of Jussieu 6 (2007), 579-611. 
[20] E.A. Moskovchenko and V.P. Kotlyrov, A New Riemann-Hilbert Problem in a Model of Stimulated Raman Scattering. — J. Phys. A: Math. Gen. 39 (2006), 14591-14610.

[21] L.D. Fadeev and L.A. Takhtadjan, Hamiltonian Methods in the Theory of Solitons. Springer, Berlin, 1987.

[22] P. Deift, Orthogonal Polynomials and Random Matrices: A Riemann-Hilbert Approach. CIMS NY University, 1999.

[23] X. Zhou, The Riemann-Hilbert problem and inverse scattering. - SIAM J. Math. Anal. 20 (1989), 966-986.

[24] G. Litvinchuk and I. Spitkovskii, Factorization of Measurable Matrix Functions. Birkhäuser-Verlag, Basel, 1987. 